\title{
Frequency of Pseudophakic Glaucoma in a Tertiary Care Hospital of Pakistan
}

\author{
Uzma Fasih ${ }^{1}$, Erum Shahid ${ }^{2}$, Arshad Shaikh ${ }^{3}$ \\ ${ }^{1-3}$ Department of Ophthalmology, Abbasi Shaheed Hospital, Pakistan
}

\begin{abstract}
Purpose: To evaluate the frequency, causes and management of pseudophakic glaucoma among the pseudophakic patients presenting in a tertiary care hospital of Pakistan.

Study Design: This was a descriptive cross sectional study.

Place and Duration of Study: Department of Ophthalmology, Abbasi Shaheed Hospital, Pakistan, from August 2017 to June 2018.

Material and Methods: Adult patients between 50 to 70 years of age with pseudophakic glaucoma were included in the study by non-probability convenience sampling after institutional review board approval. Patients with primary open angle, primary angle closure, traumatic glaucoma, diabetes mellitus and hypertension were excluded. Pseudophakic glaucoma was labeled in case of cataract surgery with intraocular lens implantation and intraocular pressure $>21 \mathrm{mmHg}$ or more in one eye along with glaucomatous optic disc or retinal nerve fiber layer defect on OCT (optical coherence tomography). Frequencies were computed for categorical variables. Data was analyzed on SPSS version 20.
\end{abstract}

Results: Twenty-eight eyes with pseudophakic glaucoma were studied. There were 15 (53.57\%) males. Mean age was $63 \pm 10.4$ SD years. Mean IOP was $30.78 \pm 7.5 \mathrm{~mm} \mathrm{Hg}$. Patients with extracapsular cataract extraction were $18(64.2 \%)$ and $10(35.8 \%)$ had phacoemulsification. Most frequent cause was posterior capsular rupture $(n=16,57.1 \%)$ followed by pupillary block, $(n=4,14.2 \%)$ and UGH $(n=3,10.7 \%)$. Medical treatment was successful in $20(71.4 \%)$ and surgical treatment was done in 8 patients.

Conclusion: Most common causes of pseudophakic glaucoma are posterior capsular rupture, vitreous loss, uveitis and pupillary block. Pseudophakic glaucoma is more common with anterior chamber intraocular lenses and extracapsular cataract extraction.

Key Words: Anterior chamber intraocular lens, Extracapsular cataract extraction, Glaucoma, posterior chamber intraocular lens, Pseudophakic glaucoma.

How to Cite this Article: Fasih U, Shahid E, Shaikh A. Frequency of Pseudophakic Glaucoma in a Tertiary Care Hospital of Pakistan, Pak J Ophthalmol. 2020;36 (1): 13-18.

DOI: https://doi.org/10.36351/pjo.v36i1.903

\section{INTRODUCTION}

Glaucoma is the second leading cause of blindness worldwide $^{1}$. It affects 60 million people globally and is responsible for $12 \%$ of global blindness. ${ }^{2}$ The term

Correspondence to: Uzma Fasih

Associate Professor Eye Department Abbasi Shaheed Hospital, Karachi Medical \& Dental College.

Email: yousufuzma@hotmail.com 'pseudophakic glaucoma' refers to the development of glaucoma following cataract surgery with Implantation of intraocular lens. It may present immediately after cataract surgery within few hours or present few weeks to months later. Pseudophakia is not directly responsible for development of glaucoma but multiple mechanisms play role in commencing glaucoma, like anterior chamber distortion, hemorrhage, inflammation, pigment dispersion, vitreous in anterior chamber, 
pupillary block, malignant glaucoma and Nd: YAG (Neodymium-doped Yttrium Aluminum Garnet) laser capsulotomy $^{3,4}$.

Incidence of Pseudophakic glaucoma differs widely across the globe. Various studies have reported the incidence of pseudophakic glaucoma from $5-41 \%$ in complicated surgeries ${ }^{5}$. Prevalence of chronic pseudophakic glaucoma has been reported between 2.1-4\% after a standard extracapsular cataract surgery and $11.3 \%$ in secondary anterior chamber implants ${ }^{5}$.

Cataract surgery is associated with postoperative inflammation. Outpouring of inflammatory cells in anterior chamber after surgical trauma leads to obstruction of trabecular meshwork. This is followed by increase in intra ocular pressure (IOP) causing glaucoma. Rupture of posterior capsule and retained lens matter may be another cause for pseudophakic glaucoma. Viscoelastic substances like sodium hyaluronate and methylcellulose used, during cataract surgery to protect the corneal endothelium and maintain the depth of anterior chamber, might lead to transient obstruction of trabecular meshwork and rise of postoperative intraocular pressure ${ }^{6}$.

Sulcus implanted posterior chamber intraocular lenses, malpositioned, and improperly sized anterior chamber intraocular lenses result in uveitis-glaucomahyphema syndrome (UGH) ${ }^{7}$. Pseudophakic pupillary block glaucoma is also seen in anterior chamber IOL (intra ocular lenses) due to vitreous humor in anterior chamber or formation of peripheral anterior synechiae. YAG laser capsulotomy for posterior capsular opacification is associated with transient rise in intraocular pressure and may become a chronic problem ${ }^{6}$.

The pseudophakic glaucoma can be diagnosed by taking a proper history regarding the cataract surgery and thorough examination including slit lamp examination, Applanation tonometry, Gonioscopy, Fundoscopy, Perimetry and OCT (optical coherence tomography) ${ }^{6}$. Management of pseudophakic glaucoma depends on the mechanism by which it is caused ${ }^{6}$.

Pseudophakic glaucoma is sometimes misdiagnosed and mismanaged. A thorough postoperative examination in this context would help in timely diagnosis and management of the disease. A thorough literature search has been done on various search engines including PubMed, Medscape, Science of web, Elsevier, Scopus and Pakmedinet but no relevent local study has been found on pseudophakic glaucoma. Causes of pseudophakic glaucoma have been addressed independently in different studies but few presented them collectively ${ }^{3,4}$. In addition, few studies have been done on pseudophakic glaucoma among children but causes among adults are not dealt with ${ }^{8}$.

We conducted this study to evaluate the frequency, causes and management of pseudophakic glaucoma among the pseudophakic patients presenting in an eye OPD of a tertiary care hospital of Pakistan.

\section{MATERIAL AND METHODS}

This was a descriptive cross sectional study carried out in an Eye OPD of Abbasi Shaheed Hospital, from August 2017 to June 2018. Sample size was calculated using Open Epi sample size calculator version 3 for demographic studies. Keeping confidence interval at $95 \%$, margin of error $5 \%$ and the hypothesized frequency $\mathrm{P}$ for the pseudophakic glaucoma 17, the sample size calculated was 213 . The study adheres to the tenets of Declaration of Helsinki. Patients were selected through nonprobability consecutive sampling technique. We included pseudophakic patients between 50-70 years of age with anterior chamber and posterior chamber intraocular lens implantation with minimum 6 weeks to maximum 5 years post operative period. Patients with history of primary open angle glaucoma, primary angle closure glaucoma, traumatic glaucoma, inflammatory glaucoma, congenital glaucoma, pseudoexfoliation, chronic uveitis, corneal opacities, aphakia, hypertension, diabetes and patients using topical or oral steroids were excluded.

A detailed history of the patients was taken. An ocular examination was carried out. Visual acuity was assessed on Snellen's chart. Slit lamp examination was done to rule out corneal edema, presence of cells or flare in anterior chamber, vitreous in anterior chamber, anterior chamber or posterior chamber IOL. Applanation tonometry was done to measure intra ocular pressure (IOP). Angle was assessed with the help of Gonioscopy. A detailed fundoscopic assessment of the disc and RNFL was done. Perimetry and OCT (optical coherence tomography) were further done to establish the diagnosis of glaucoma. Pseudophakic glaucoma was labeled when the patient had history of cataract surgery with intraocular lens implantation (anterior chamber IOL/posterior chamber IOL) and intraocular pressure greater than $21 \mathrm{~mm} \mathrm{Hg}$ 
or more in one eye along with glaucomatous optic disc (vertical cup to disc ratio $0.7 \pm 0.2$ ) and retinal nerve fiber layer defect on OCT or typical glaucomatous field defects on perimetery. Patients were managed according to the mechanism of glaucoma either medically or surgically or by laser treatment (argon laser trabeculoplasty or YAG laser iridotomy). As the post operative duration for inclusion criteria was from 6 weeks to 5 years post operatively, the topical steroids had been stopped by that time and if they had to be prescribed for postoperative inflammation the patients were monitored for IOP and responders were excluded.

Data was collected and analyzed on SPSS version 20. Frequencies were computed for categorical variables like gender, causes and management of pseudophakic glaucoma. Means with standard deviation SD were calculated for age, intraocular pressure and duration of cataract surgery.

\section{RESULTS}

Total number of pseudophakic patients in this study was 213. Among them $28(13.1 \%)$ patients were diagnosed with pseudophakic glaucoma. There were $15(53.5 \%)$ male patients. Mean age was $63 \pm 10.4$ years. Mean IOP was $30.78 \pm 7.5 \mathrm{~mm} \mathrm{Hg}$. Eighteen patients $(64.2 \%)$ underwent extracapsular cataract extraction and $10 \quad(35.8 \%)$ underwent phacoemulsification. There were $7(25.0 \%)$ patients with anterior chamber intraocular lens and 21 (75.0\%) patients with posterior chamber intraocular lens. Mean duration of presentation after cataract surgery was 2.7 \pm 2.6 years. Other demographic features of glaucoma patients at one year follow up are given in table 1 .

Most frequent cause of pseudophakic glaucoma was posterior capsular rupture at the time of surgery $(\mathrm{n}=16,57.1 \%)$. This was followed by pupillary block $(\mathrm{n}=4,14.2 \%)$ and $\mathrm{UGH}(\mathrm{n}=3,10.7 \%)$. Less common causes were YAG laser capsulotomy $(\mathrm{n}=2,7.1 \%)$ and retained lens matter $(n=1,7.1 \%)$. Frequencies of other causes of pseudophakic glaucoma are given in table 2 . Medical treatment was successful in $20(71.4 \%)$ cases followed by surgical treatment (trabeculectomy in 4 , $14.2 \%)$, and peripheral iridotomies in 4 (14.2\%) patients for control of intraocular pressure as shown in table 3 .
Table 1: Demographic characteristic of the patients.

\begin{tabular}{ll}
\hline Variables & Frequency $(\%)$ \\
\hline Pseudophakic glaucoma & $28(13.1 \%)$ \\
Mean Age & $63 \pm 10.4$ years SD \\
Male & $15(53.5 \%)$ \\
Female & $13(46.5 \%)$ \\
Mean IOP & $30.78 \pm 7.5 \mathrm{~mm} \mathrm{Hg} \mathrm{SD}$ \\
Mode of Surgery & $18(64.2 \%)$ \\
ECCE & $10(35.8 \%)$ \\
Phacoemulsification & $7(25.0 \%)$ \\
Type of Intra Ocular Lens & $21(75.0 \%)$ \\
AC IOL & $2.7 \pm 2.6$ years SD \\
PC IOL & \\
Mean Duration of surgery & \\
\hline
\end{tabular}

IOP: intra ocular pressure

AC IOL: anterior chamber intra ocular lens

PC IOL: posterior chamber intra ocular lens

ECCE: extra capsular cataract surgery

Table 2: Causes of pseudophakic glaucoma.

\begin{tabular}{llc}
\hline Causes of pseudophakic glaucoma & Frequency n (\%) \\
\hline - & Posterior capsular & $16(57.1 \%)$ \\
- $\quad$ rupture + Uveitis & \\
- & UGHillary block & $4(14.2 \%)$ \\
- & YAG laser capsulotomy & $3(10.7 \%)$ \\
- & Retained lens matter & $2(7.1 \%)$ \\
- & Pigment dispersion & $1(3.5 \%)$ \\
- & Malignant glaucoma & $1(3.5 \%)$ \\
\hline
\end{tabular}

Table 3: Management of Pseudophakic Glaucoma.

\begin{tabular}{lc}
\hline Treatment Modality & Frequency n (\%) \\
\hline Medical Treatment & $20(71.4 \%)$ \\
Trabeculectomy & $4(14.2 \%)$ \\
Peripheral Iridectomy & $4(14.2 \%)$ \\
\hline
\end{tabular}

\section{DISCUSSION}

The term pseudophakic glaucoma refers to the glaucoma, which is seen after the cataract surgery with implantation of intraocular lens. There may be any one or multiple mechanisms working together for this entity ${ }^{9}$.

We studied 213 pseudophakic patients and pseudophakic glaucoma was diagnosed in 28 (13.1\%) patients. A study conducted in the same center in 2007 for evaluation and management of secondary glaucoma, reported $31(29.2 \%)$ patients with pseudophakic glaucoma, 21 with posterior chamber intraocular lens and 10 with anterior chamber intraocular lens ${ }^{10}$. They had reported a higher frequency as their sample size specifically consisted of 
secondary glaucoma patients. Chennai glaucoma study reported a prevalence of aphakic/pseudophakic glaucoma in $9.77 \%$ patients in rural India and $9.36 \%$ in their study in urban parts of India ${ }^{11}$. Another study from Andhra Pardesh reported a prevalance of $14.6 \%$ which is quite similar to our study ${ }^{12}$.

In South Africa a prevalence of $0.02-0.04 \%$ is reported $^{13}$. Another study from Thailand reported no case of pseudophakic glaucoma following cataract surgery ${ }^{14}$. These figures display a drastic difference in frequency of pseudophakic glaucoma between India, Pakistan and other developed countries. This disparity could be due to the fact that different cataract eradication programs including camp surgeries have been running in our part of the world but unfortunately quality and standard is still questionable. Phacoemulsification is the procedure of choice for cataract removal in developed countries, which is associated with fewer complications.

Mean age of patients in our study was $63 \pm 10.4$ $\mathrm{SD}$ years. Arvind et al have reported a mean age of $64.85 \pm 8.74$ years, which is quite similar to our study. They have also suggested that increasing age could also be a risk factor for pseudophakic glaucoma ${ }^{9}$. Mean intraocular pressure reported in our study was $30.78 \pm 7.5 \mathrm{~mm} \mathrm{Hg}$. Arshinoff et al reported a mean IOP of $22.2 \mathrm{~mm} \mathrm{Hg}$ in their study ${ }^{15}$.

In our study, Pseudophakic glaucoma was more frequent among patients with ECCE than phacoemulsification. Prevalence of pseudophakic glaucoma was reported in $2.1-4 \%$ patients in a study by Park et al, after standard extracapsular cataract extraction ${ }^{16}$. Cinoti has also reported an increased incidence $(7.5 \%)$ of glaucoma after $\mathrm{ECCE}^{17}$. In developing countries, patients are still presenting with mature and hypermature cataracts, which are dealt with extra capsular cataract surgery or small incision cataract surgery. High frequency of glaucoma in our setup could be due to the fact that our hospital is a tertiary care hospital. Patients from other primary and secondary centers are being referred, particularly the complicated cases. Surgeries are also sometimes performed by trainees. This again has high surgical complication rate.

Posterior Chamber Intraocular lens implant in our study was seen in $21(75.0 \%)$ patients while Anterior Chamber Intraocular lens implant was reported in 10 (25.0\%) patients. A study by Stark has also reported an incidence of $5.5 \%$ rise in intraocular pressure in anterior chamber intraocular lens implantation as compared to that of posterior chamber intraocular lens implantation $(1.6 \%)^{18}$. Ang et al has reported a rise of $30 \mathrm{~mm} \mathrm{Hg}$ in IOP in $20 \%$ of the patients following posterior capsular rupture during phacoemulsification even after 24 hours of the surgery. More than $50 \%$ of his patients required anterior vitrectomy ${ }^{19}$. Both openangle and closed-angle glaucoma may present in the early as well as late postoperative period. Open-angle glaucoma may result from blockage of trabecular meshwork by vitreous, retained nuclear/cortical fragments, inflammatory cells, and pigment dispersion due to excessive intraocular manipulation ${ }^{20}$.

Postoperative uveitis along with posterior capsule rupture is one of the common causes of glaucoma among pseudophakic patients. Diverse causes of uveitis that lead to glaucoma after cataract surgery are vitreous in anterior chamber following posterior capsular rupture, pigment dispersion, retained lens matter, retained viscoelastic and hyphema ${ }^{3,4}$. Patients who presented with glaucoma in association with posterior capsular rupture were managed medically by topical beta blockers, topical and systemic carbonic anhydrase inhibitors and topical steroids and nonsteroidal anti-inflammatory drops. Topical beta blockers and carbonic anhydrase inhibitors should be the preferred choice and miotics and prostaglandin inhibitors should be avoided ${ }^{21}$.

Pupillary block glaucoma was encountered in 4 (14.2\%) patients. All of them were treated medically with beta Blockers, carbonic anhydrase inhibitors and oral carbonic anhydrase inhibitors. Later on iridectomy along with pupilloplasty was done when the corneal edema was clear.

UGH syndrome caused glaucoma in $3(10.7 \%)$ patients in this study. All of these patients had anterior chamber lens implantation (AC IOL). UGH syndrome also known as Ellingson syndrome is generally caused by a subluxated or malposition anterior or posterior chamber intraocular lens. It causes mechanical trauma to the adjacent structures as iris or cilliary body. As a result, there may be persistent inflammation, micro hyphema, pigment dispersion and rise in intraocular pressure $^{22}$. In this group, all patients had to go for trabeculectomy while one patient required removal of AC IOL. A study from china had reported 9.1\% UGH syndrome, in which intraocular lenses had to be removed $^{22}$.

Nd: YAG laser capsulotomy for posterior capsular 
opacification is often accompanied with transient rise of intraocular pressure due to blockage of trabecular meshwork by inflammatory cells. It may become a chronic problem ${ }^{6}$. Presentation of glaucoma following YAG laser capsulotomy was in $2(7.1 \%)$ patients. Both were medically managed.

One $(3.5 \%)$ patient presented with glaucoma associated with retained lens matter. Glaucoma associated with retained lens matter is an open angle glaucoma caused by the obstruction of outflow by macrophages and inflammatory mediators. In addition, there may be precipitation of lens proteins in trabecular meshwork ${ }^{6}$. This patient was treated medically by topical beta blockers, topical and oral carbonic anhydrase inhibitors and topical steroids.

Glaucoma due to pigment dispersion was seen in 1 (3.5\%) patients. Mierlo et al had reported a case series of 3 patients with pigment dispersion. Improperly sulcus implanted posterior chamber intraocular lenses cause iatrogenic insult ${ }^{23}$. These patients had to undergo trabeculectomy after failure of medical treatment.

Viscoelastic substance causes $55-60 \%$ reduction in aqueous drainage when injected in anterior chamber. Arshinoff et al had reported that dispersive viscoelastics are more helpful in protecting the ocular structures like corneal endothelium but are difficult to remove from the anterior chamber as compared to the cohesive ones ${ }^{16}$. They also reported that retained viscoelastic might be one of the main factors for postoperative rise of IOP more than $21 \mathrm{~mm} \mathrm{Hg}^{16}$. Fortunately, no case of retained viscoelastic substance was encountered in our study.

Malignant glaucoma presented in 1 (3.5\%) patient in our study who was a hypermetrope. This is a rare entity, which is frequently encountered after trabeculectomy in narrow angle glaucoma but rarely can be seen after cataract surgery with intraocular lens implantation ${ }^{24}$. Our patient responded well to medical treatment, which included cycloplegic, steroid and pressure lowering agents.

We found carbonic anhydrase inhibitors alone or in combination with beta blockers to be more effective for long term control of IOP in pseudophakic glaucoma. Ermis et al also found carbonic anhydrase inhibitors more effective for long term reduction of $\mathrm{IOP}^{25}$.

Strength of our study is that it has thoroughly investigated into the causes of pseudophakic glaucoma. It represents local data and will be helpful in managing patients in our region. This data can be used to generate hypothesis. Further case control or cohort studies can be carried out in future to see association with variables. However, Pseudophakic glaucoma should be thoroughly investigated and managed accordingly. Postoperative care of such patients will significantly improve the quality of vision and life. Limitation of our study is short follow up period of one year.

\section{CONCLUSION}

Anterior chamber intraocular lens is more frequently associated with pseudophakic glaucoma than sulcus implanted posterior chamber lens. Chances of Pseudophakic glaucoma are more in patients who undergo ECCE as compared to phacoemulsification. Majority of pseudophakic glaucoma responded well to medical treatment while others to surgical treatment.

\section{Ethical Approval}

The study was approved by the Institutional review board/Ethical review board.

\section{Conflict of Interest}

Authors declared no conflict of interest

\section{Authors' Designation and Contribution}

Uzma Fasih; Associate Professor: Study design, manuscript writing, literature review.

Erum Shahid; Assistant Professor: Literature review and final review.

Arshad Shaikh; Professor: Literature review and final review.

\section{REFERENCES}

1. Kumarasamy NA, Lam FS, Wang AL, Theoharides TC. Glaucoma: Current and developing concepts for inflammation, pathogenesis and treatment. Eur J Inflam. 2006 Sep; 4 (3): 129-37.

2. Dineen B, Bourne RR, Jadoon Z, Shah SP, Khan MA, Foster A, Gilbert CE, Khan MD. Causes of blindness and visual impairment in Pakistan. The Pakistan national blindness and visual impairment survey. Br J Ophthalmol. 2007 Aug. 1; 91 (8): 1005-10.

3. Tomey KF, Traverso CE. The glaucomas in aphakia and pseudophakia. Surv Ophthalmol. 1991 Sep. 1; 36 (2): 79-112. 
4. Lee LC, Pasquale LR. Surgical management of glaucoma in pseudophakic patients. Semin Ophthalmol. 2002; 17(3-4):131-7

5. Yi K, Chen TC. Aphakic glaucoma after congenital cataract surgery. Intern Ophthalmol Clin. 2008; 48 (2): 87-94.

6. Shields MB, Rand R.. Allingham, Karim F.. Damji. Shields' textbook of glaucoma. Chapter 26 Lippincott Williams \& Wilkins; 2005.

7. Zhang L, Hood CT, Vrabec JP, Cullen AL, Parrish EA, Moroi SE. Mechanisms for in-the-bag uveitisglaucoma-hyphema syndrome. J Cat Ref Surg. 2014; 40 (3): 490-2.

8. Kirwan C, Lanigan B, O'Keefe M. Glaucoma in aphakic and pseudophakic eyes following surgery for congenital cataract in the first year of life. Acta Ophthalmologica. 2010; 88 (1): 53-9.

9. Arvind H, George R, Raju P, Ramesh SV, Baskaran M, Paul PG, McCarty C, Vijaya L. Glaucoma in aphakia and pseudophakia in the Chennai Glaucoma Study. Br J Ophthalmol. 2005; 89 (6): 699-703.

10. Fasih U, Fehmi MS, Shaikh N, Shaikh A. Secondary glaucoma - causes and management. Pak J Ophthalmol. 2008; 24 (2): 86-92.

11. George R, Arvind H, Baskaran M, Ramesh SV, Raju P, Vijaya L. The Chennai glaucoma study: prevalence and risk factors for glaucoma in cataract operated eyes in urban Chennai. Indian J Ophthalmol. 2010; 58 (3): 243.

12. Dandona L, Dandona R, Srinivas M, Mandal P, John RK, McCarty CA, Rao GN. Open-angle glaucoma in an urban population in southern India: the Andhra Pradesh eye disease study. Ophthalmology. 2000; 107 (9): 1702-9.

13. Rotchford AP, Johnson GJ. Glaucoma in Zulus: a population-based cross-sectional survey in a rural district in South Africa. Archives Ophthalmol. 2002; 120 (4): 471-8.

14. Bourne RR, Sukudom P, Foster PJ, Tantisevi V, Jitapunkul S, Lee PS, Johnson GJ, Rojanapongpun P. Prevalence of glaucoma in Thailand: a population based survey in Rom Klao District, Bangkok. Br J Ophthalmol. 2003; 87 (9): 1069-74.

15. Arshinoff SA, Albiani DA, Taylor-Laporte J. Intraocular pressure after bilateral cataract surgery using Healon, Healon5, and Healon GV. J Cat Ref Surg. 2002; 28 (4): 617-25.

16. Kurimoto Y, Park M, Sakaue H, Kondo T. Changes in the anterior chamber configuration after smallincision cataract surgery with posterior chamber intraocular lens implantation. Am J Ophthalmol. 1997; 124 (6): 775-80.

17. Cinotti AA, Jacobson JH. Complications Following Cataract Extractions*: An Analysis of 1,001 Cataract Operations. Am J Ophthalmol. 1953; 36 (7): 929-36.

18. Stark WJ, Worthen DM, Holladay JT, Bath PE, Jacobs ME, Murray GC, et al. The FDA report on intraocular lenses. Ophthalmology, 1983; 90 (4): 311-7.

19. Ang GS, Whyte IF. Effect and outcomes of posterior capsule rupture in a district general hospital setting. J Cat Ref Surg. 2006; 32 (4): 623-7.

20. Chakrabarti A, Nazm N. Posterior capsular rent: Prevention and management. Indian $\mathbf{J}$ Ophthalmol. 2017; 65 (12): 1359.

21. Zemba M, Camburu G. Uveitis-glaucoma-hyphaema syndrome. General review. Romanian J Ophthalmol. 2017; 61 (1): 11.

22. Chan TC, Lok JK, Jhanji V, Wong VW. Intraocular lens explantation in Chinese patients: different patterns and different responses. Int Ophthalmol. 2015; 35 (5): 679-84.

23. Mierlo VC, Pinto LA, Stalmans I. Surgical management of iatrogenic pigment dispersion glaucoma. J Curr Glaucoma Pract. 2015; 9 (1): 28.

24. Reed JE, Thomas JV, Lytle RA, Simmons RJ. Malignant glaucoma induced by an intraocular lens. Ophthalmic Surgery, Lasers and Imaging Retina. 1990; 21 (3): 177-80.

25. Ermis SS, Ozturk F, Inan UU. Comparing the effects of travoprost and brinzolamide on intraocular pressure after phacoemulsification. Eye. 2005; 19 (3): 303. 